$39^{\text {th }}$ AIAA/ASME/SAE/ASEE

AIAA-2003-5095

Joint Propulsion Conference and Exhibit

Von Braun Center, Huntsville, Alabama

July 20-23, 2003

\title{
EXPECTED DEPLOYMENT DYNAMICS OF PRoSEDS
}

E.C. Lorenzini

Harvard-Smithsonian

Center for Astrophysics

Cambridge, MA 02138

Email: elorenzini@acfa.harvard.edu

M.L. Cosmo

Harvard-Smithsonian

Center for Astrophysics

Cambridge, MA 02138

Email: mosmo@cfa.harvard edu

\author{
K. Welzyn \\ Mail Code TD55 \\ NASA Marshall Space Flight Center \\ Marshall Space Flight Center, AL 35812 \\ Email: ken.welzyn@msfc.nasa.gov
}

\begin{abstract}
*
The control law for ProSEDS deployment is a modification of the control routine that was successfully used in the flight of SEDS-II. Unlike SEDS, the tether of ProSEDS consists of different sections with different mechanical characteristics. A non-linear control trajectory in phase-space (i.e., the reference profile) is fed forward to the controller to guide the satellite, at the tether tip, to the desired final state under nominal conditions and no external perturbations. A linear feedback control is applied by the brake to keep the actual trajectory as close as possible to the reference. The paper also shows the results of simulations of deployment dynamics with and without noise. The control law has thus far been developed and tested on the ground for the original ProSEDS tether configuration of $15 \mathrm{~km}$. A new reference will have to be designed and tested for other tether configurations.
\end{abstract}

\section{INTRODUCTION}

The Propulsive Small Expendable Deployment System (ProSEDS) will be a demonstration flight of a new anode design for electrodynamic tethers (EDTs). The system is much lighter than the predecessor electrodynamic tethered satellite

* Copyright (C) 2003 by the American Institute of Aeronautics and Astronautics, Inc. The U.S. Government has a royalty-free license to exercise all rights under the copyright claimed herein for Governmental purposes. All other rights are reserved by the copyright owner. system (TSS) yet ProSEDS is expected to draw a current that is about four times higher. The high current acting on a light tether system will make it more susceptible to dynamic instabilities driven by electrodynamic forces than the heavier systems of the past. Reference ${ }^{1}$ states that the stability of an electrodynamic tether system diminishes with the increase of the ratio $\mathrm{R}=\mathrm{F}_{\mathrm{ey}} / \mathrm{F}_{\mathrm{g} z}$ where $\mathrm{F}_{\mathrm{cy}}$ is the outof-the- orbital-plane (out-of-plane) component of the electrodynamic force and $F_{\mathrm{gz}}$ is the vertical component of the gravity gradient force. Typically, the ratio $R$ is smaller than one in an EDT. The electrodynamic forces produce a weak instability consisting in a slow growth of the amplitude of the 3-dimensional libration of the tether system. The amplitude will take a longer time to grow if $R$ is small and the system starts from a null or small libration amplitude, that is, with the tether close to the local vertical.

The ProSEDS mission is relatively short and, consequently, the stability issue is much less serious than for a long-duration mission. Nevertheless, the resulting oscillations will be smaller and the stability margin larger if the initial libration is small. The requirement for ProSEDS is an initial libration with an upper limit of $20^{\circ}$. The goal of the deployment control strategy is to make that libration as small as possible within that upper limit.

The ProSEDS deployment control law follows in part the control strategy used successfully to deploy SEDS- $\mathrm{II}^{2}$. The ProSEDS tether, however, is different from the SEDS tether. The ProSEDS tether is made of sections with different mechanical 
characteristics and special attention must be paid to the deployment of the electrodynamic portion of the tether as shown later on. In the following, we will describe the control strategy and then apply the control law to the original tether configuration of ProSEDS for which the control law ha been so far developed and tested on the ground.

\section{CONTROL LAW STRATEGY}

ProSEDS utilizes the same deployment hardware of SEDS. The deployer, built by Tether Applications, had two successful flights in the 1990s. It is a light deployer of simple and clever design ${ }^{3}$. However, its sensors were not designed to be used in a close-loop control scheme. The sensors in question are a couple of photocells that, working together, measure the number of turns deployed from the spool. There are no sensors to measure directly the tether deployment velocity. The actuator (brake) has also a simple design and a highly non-linear response that makes the design of the control law much more challenging.

The goal of the control law is to control a nonlinear plant, both in terms of dynamics and actuator response, in the presence of uncertainties affecting the actuator and the plant. The performance goals are: (1) a libration at the end of deployment well within the $20^{\circ}$ limit; (2) a fully deployed tether without any significant number of coils left in the deployer; and (3) a small velocity $(<2 \mathrm{~m} / \mathrm{s})$ at the end of deployment to avoid a strong tension spike. One additional requirement calls for the activation of the brake only after the first kilometer of tether has been deployed because, since the tether tension is too low at short tether lengths, an over braking could stop the momentum and halt deployment at short range.

\section{Closed-loop portion}

The control law utilizes the input-output linearization technique ${ }^{4}$ to eliminate the non linearities by means of a non-linear solution of the nominal controlled response as explained in the following. The strategy adopted for deriving the control law divides the control problem into two parts: (1) finding the solution of a two point boundary value problem; and (2) developing a local linear feedback controller.

The solution of the boundary value problem, obtained through an optimization process, provides the reference length $L_{r e f}$ and velocity $\dot{L}_{r e f}$ profiles and an associated reference brake profile $B_{\text {ref }}$ (i.e., the reference control input). A brief description of the parametric optimization is provided in the following (see Ref. I for a more detailed explanation).

A two-dimensional (in the orbital plane) model is utilized for deriving the reference profile as follows:

$$
\begin{aligned}
& \ddot{\vartheta}+2 \frac{\dot{L}}{L}(\dot{\vartheta}-\Omega)+\frac{3}{2} \Omega^{2} \sin (2 \vartheta)=0 \\
& \ddot{L}-L\left[(\dot{\theta}-\Omega)^{2}+\Omega^{2}\left(3 \cos ^{2}(\theta)-1\right)\right]=-T / m
\end{aligned}
$$

where $L$ is the tether length, $\theta$ the in-plane libration angle, $T$ the tether tension and $m$ is the reduced mass of the tethered system. Eqns. (1) are the wellknown dumbbell equation for a tethered system with the assumptions of circular orbit, massless tether, point end-bodies, and negligible external perturbations.

The tension model for the SEDS deployer is as follows 5 :

$$
T=\left[T_{0}+I \rho \dot{L}^{2} A_{r e l}^{-E}\right] \cdot e^{B} \cdot e^{f \cdot|1 .| 3-\vartheta \mid}
$$

where $A_{r e l}=1-A L / L_{\text {end }}, L_{\text {end }}=$ final tether length, $A=$ annulus solidity, $E=$ area exponent, $B=$ brake parameter $=2 \pi f n$ with $n=$ number of brake turns, $f$ $=$ friction coefficient, $T_{0}=$ static (or minimum) tether tension, $I=$ inertia multiplier, $\rho=$ linear density of tether, $\theta=$ tether's exit angle (for a deployer aligned along the Nadir $\theta$ coincides with the in-plane libration angle of the tether with respect to $\mathrm{LV}$ ).

The optimization is carried out by parameterizing the function $B$ with $k$ cubic splines identified by the $k$ values at their anchor points. A cost function $F$ is then defined as a quadratic function of the error values between the actual and the desired components of the state vector at the end of deployment. Additional mission-related constraints are added to the cost function such as the minimum allowable velocity during deployment (see Ref. 1).

The optimization routine ${ }^{6}$ first makes a trial selection of the $k$ brake parameters in a bounded hyper-volume of $k$ dimensions. Subsequently, a numerical integrator is utilized to compute the final state of the system for every iteration and, hence, the cost function $F$. Based on the variational trend of the cost function from one iteration to the next, the optimization routine selects successive sets of values for the brake parameters $B_{k}$ till the cost 
function is minimized. The process typically takes about 300-500 iterations to converge.

Once the reference profile is available, the control problem is reduced to designing a controller for a linear-time-varying (LTV) system. The locally-linear feedback control in our case is a PD controller which drives the system onto the reference length and velocity profiles by canceling the length and velocity errors with respect to the reference. In summary, the control law consists of the feed-forward input profile $B_{r e f}$ and the feedback controller $u$ as follows:

$$
B=B_{r e f}+u
$$

where

$$
u=k_{1} \delta L+k_{2} \delta \dot{L}
$$

is the linear feedback with $\mathrm{k}_{1}$ and $\mathrm{k}_{2}$ the control gains and $\delta L$ and $\delta \dot{L}$ the length and velocity errors with respect to the reference length and velocity profiles.

In its simplest formulation the PD law of eqn. (3.2) has constant gains. One disadvantage of the constant gains, however, is that the control authority of the feedback is relatively strong at short tether lengths. Furthermore, since the control law is activated only after $t>400$ s (i.e., for $L>1$ $\mathrm{km}$ ), the tracking errors with respect to the reference trajectory may be substantial at the time of activation resulting in a sudden (and very undesirable) application of the brake at short tether length. In order to avoid these drawbacks, eqn. (3.1) has been modified by weighing the control gains with the reference brake profile $B_{\text {ref }}$ as follows:

$$
B=B_{r e f}\left(1+k_{1} \delta L+k_{2} \delta \dot{L}\right)
$$

The formulation above satisfies the requirement of reducing the control authority of the feedback at short tether lengths and enabling a gradual activation of the brake (it should be noted that Bref is a monotonously increasing function). The simple technique adopted for reducing the control authority has the essential advantage of being neither memory nor CPU intensive (two critical requirements for the flight software) as it uses a weighing function $B_{\text {ref }}$ that is available to the onboard computer.

\section{Open-loop Portion}

The tether of ProSEDS that has been tested for flight thus far consists of a $10.1-\mathrm{km}$, nonconductive Dyneema segment (attached to the satellite), followed by a $4.86-\mathrm{km}$ conductive and bare tether and a $215-\mathrm{m}$ conductive and insulated tether. The $4.86-\mathrm{km}$ conductive portion of the tether is covered by a special coating called CCOR to keep its temperature low.

The coating does not tolerate strong abrasion. Consequently, this tether segment must be deployed with very small or no braking, thus preventing the use of the closed-loop control law. Finally, the tether exit velocity, that is bound to increase during deployment of the CCOR segment, must be reduced to a small value during deployment of the last 215 $m$, insulated portion of the tether. If, as it appears likely, the tether configuration is changed, a new reference must be developed and on-ground deployment tests must be conducted. 


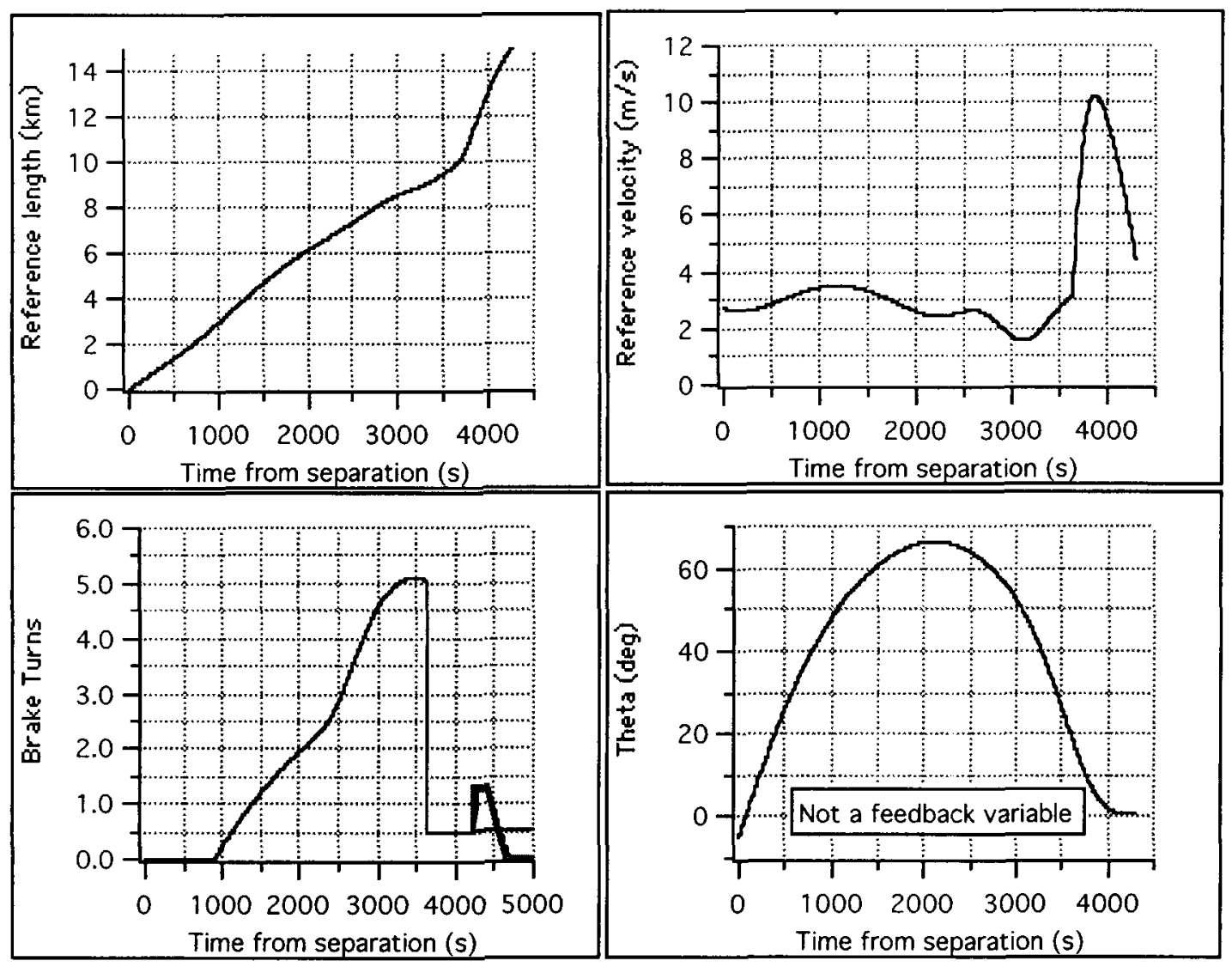

Fig. 1 Reference profiles (Ref. \#78) for ProSEDS deployment. The brake profile (thick line) also shows the slow-down profile which is actually not part of the optimization process (see text).

The open-loop portion of the control law is shaped with the goal of satisfying the following requirements: (1) limit the tether exit velocity during deployment of the CCOR tether to less than $11 \mathrm{~m} / \mathrm{s} ;$ (2) reduce the final velocity to less than 2 $\mathrm{m} / \mathrm{s}$; and (3) guarantee that close to the entire tether (with the possible exclusion of a few turns) is deployed.

The amount of brake to be applied on the CCOR tether is estimated from the measured friction characteristics of the tether. This information is provided to the optimization routine that computes the reference profile for the Dyneema and CCOR tether segments. The reference profile by itself will drive under nominal conditions the system to the desired speed, libration angle and angular rate at the end of CCOR and beginning of the insulated tether segment (last 215 $\mathrm{m}$ of tether).
The flight reference profiles for ProSEDS are shown in Figs. 1. The in-plane libration angle, also shown in this figure, is not part of the control scheme as previously explained. The reference brake profile $B_{r e f}$ is the non-linear part of the control input which is fed forward to the actuator. This non-linear part makes possible a linearization of the perturbed response of the system around the nonlinear solution. In other words the reference input $\mathrm{B}_{\text {ref }}$ is adjusted by the linear feedback in order to keep the acttual response close to the reference output response ( $L_{r e f}$ and $\left.\dot{L}_{r e f}\right)$. For this reason, this control technique is sometime called input-output linearization.

The slow down of the tether exit velocity in the last $215 \mathrm{~m}$ is accomplished with a trapezoid brake profile (shown in Fig. 1) in which the brake is ramped up quickly once the insulated portion is reached, followed by a plateau at constant brake to 
dissipate the residual kinetic energy. The final phase is a slow removal of brake to let any remaining tether coils out of the spool. The height of the plateau is chosen so that the braking force is close to the gravity gradient force (that pulls the tether out) in order to allow a slow pay out of tether. The slow-down brake profile reduces the tether exit speed from the $4.5 \mathrm{~m} / \mathrm{s}$ indicated in Fig. 1 at the end of the CCOR tether to close to zero. Note that the slow-down profile is not part of the optimization process and, consequently, the speed shown in Fig.l does not include the affect of the slow-down phase.

\section{Flight control software}

Deploying from a passive spool with a tether rubbing on various metal surfaces is dominated by the stick-slip mechanism as it was inferred from the data of the SEDS-I flight. This mechanism gives rise to an output signal from the turn counter with a high noise level. Effective filtering is, therefore, important in order to eliminate the noise components from the turn counter output signal and, consequently, compute a noise-free turn rate by means of numerical derivation.

The filter adopted in this control scheme is a recursive filter with the following formulation?

$$
\mathrm{y}(\mathrm{i}+1)=a \mathrm{y}(\mathrm{i})+\mathrm{c}[\mathrm{z}(\mathrm{i}+1)-a \mathrm{y}(\mathrm{i})]
$$

where $z(i)$ and $y(i)$ are the measured and the filtered variables, respectively, taken at the $\mathrm{i}^{\text {th }}$ step, $a$ determines the type of filtering, and $c$ determines the cut-off frequency. Specifically, for $a=1$, eqn. (5) represents a low-pass filter. The amplitude of the filtered variable is given by

$$
\mathrm{M}=\mathrm{c}\left[a^{2}(\mathrm{c}-1)^{2}+2 a(\mathrm{c}-1) \cos (\omega \tau)+1\right]^{-1 / 2}
$$

where $\tau$ is the sampling frequency of the measured variable. Specifically, for $c=0.15$, the cut-off frequency (defined as the frequency at $-3 \mathrm{~dB}$ ) is about $0.02 \mathrm{~Hz}$. A cut-off frequency of $0.02 \mathrm{~Hz}$ is adequate for ProSEDS since, from the previous flights of SEDS, the lowest-frequency noise component is at $0.03 \mathrm{~Hz}$.

The flight control software is based on an outer loop sampled at every 8 seconds and an inner loop sampled at every 1 second. The output of the turn counter (see Fig. 2 ) $^{8}$, sampled at every second, is filtered and the turn count rate is computed by taking the numerical derivative of the filtered turn counts over 8-s intervals. This process provides a smooth turn count rate despite the high noise level of the turn count signal. At every 8-s, the smoothed turn count and turn rates are compared to the reference turn count and rate for computing the feedback $u$.

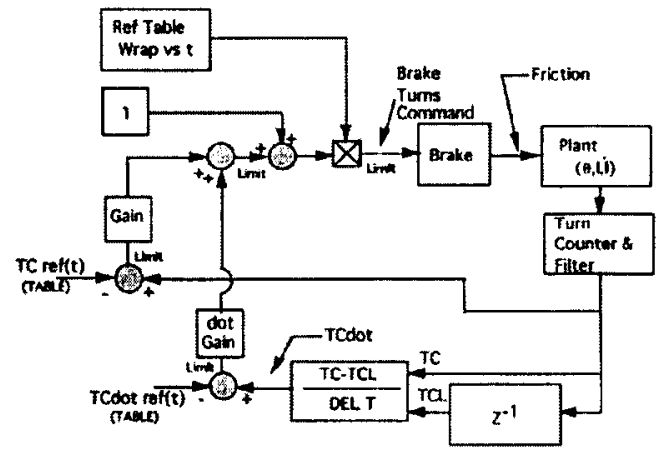

Fig. 2 Flow chart of control system [NASA/MSFC and Ref. 8]

\section{PRE-FLIGHT SIMULATIONS}

\section{Sensitivity to static tension}

The parameter with the greatest influence on the deployment dynamics is the static tension $\mathrm{T}_{0}$. A variation of the static tension during deployment affects dramatically the tether dynamics in general and the tether libration in particular.

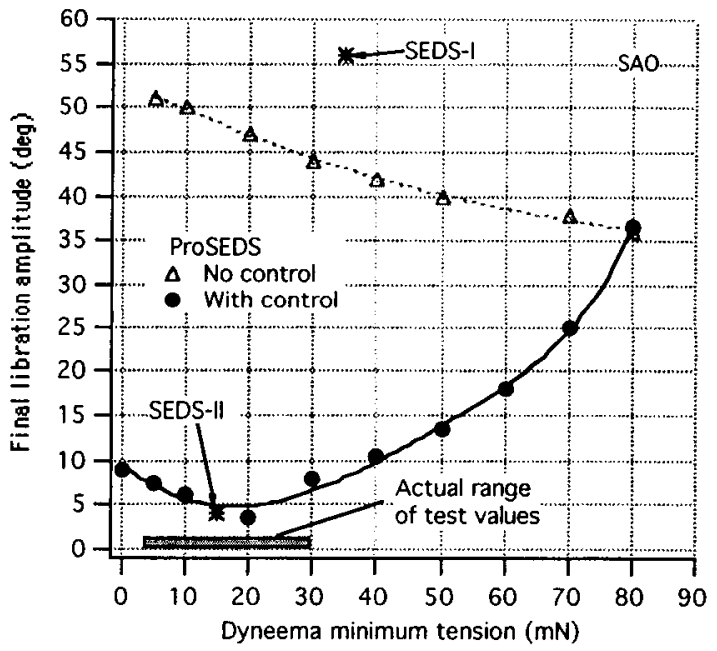

Fig. 3 Final libration amplitude of ProSEDS vs. Dyneema tether minimum tension. The performance points of SEDS-I and SEDS-II are also shown in the figure.

A value of $\mathrm{T}_{0}>80 \mathrm{mN}$ stops deployment within less than a kilometer because the initial momentum 
is dissipated before the gravity gradient is sufficiently strong to overcome the frictional forces.

Figure 4 shows the deployment dynamics with the control system activated for a tether minimum tension $\mathrm{T}_{0}=20 \mathrm{mN}$ for the Dyneema and $150 \mathrm{mN}$ for the CCOR portion of the tether, respectively.

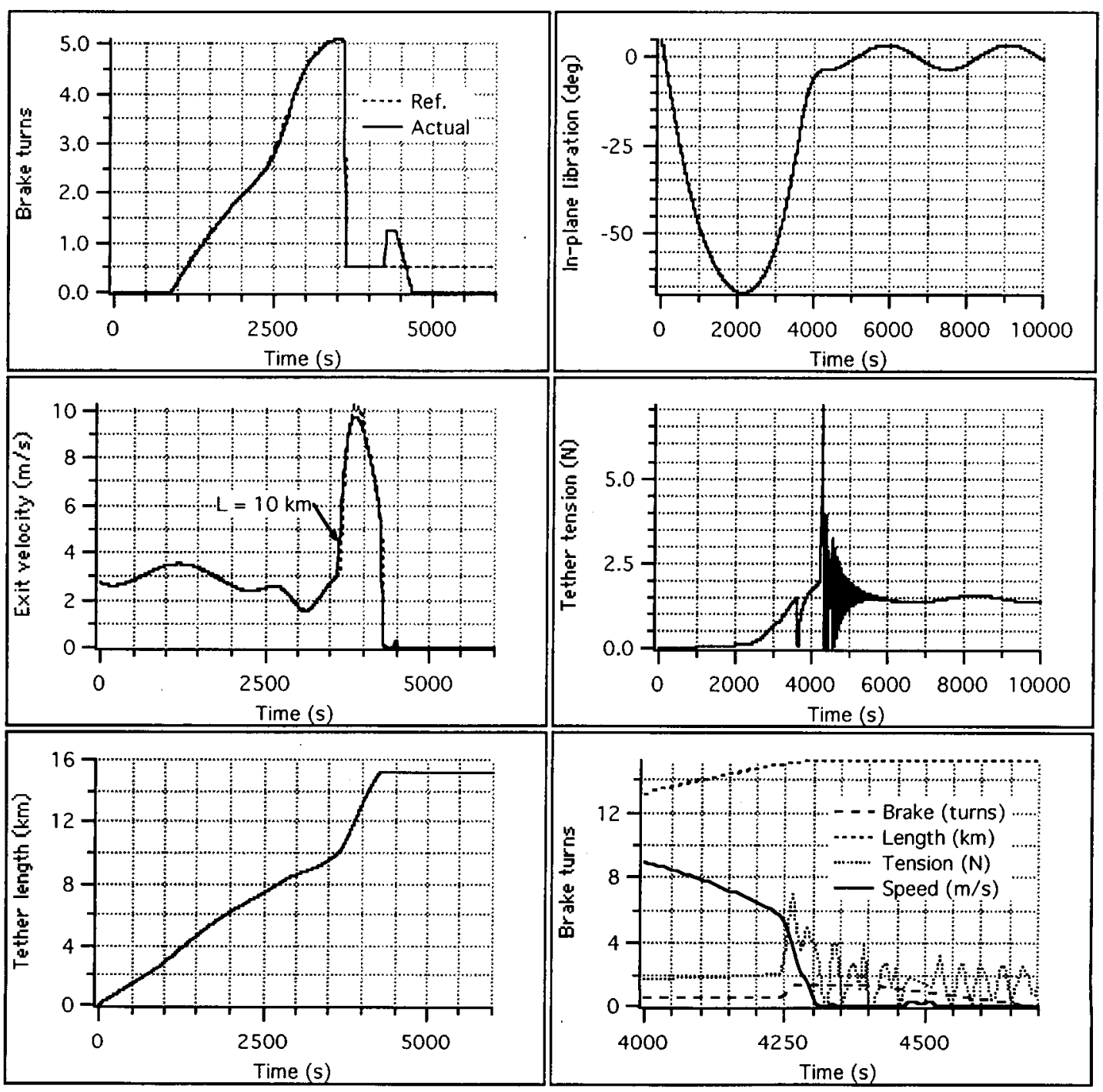

Fig. 4 Pre-flight simulation of deployment dynamics for a tether minimum tension $\mathrm{T}_{0}=20 \mathrm{mN}$ in the Dyneema and $150 \mathrm{mN}$ in the CCOR tether segments.

\section{Sensitivity to Random Noise}

In order to test the effect of broad-band random noise upon the control law, a structured noise is superimposed on the tether tension. Specifically, the structure of the tension affected by noise $T_{N}$ is as follows:

$$
T_{N}=S+N_{a}+T\left(1+N_{m}\right)
$$

where $T$ is the noise-free tether tension, $N_{a}$ and $N_{m}$ are broad-band random noise components end a $S$ is a sinusoidal low-frequency noise component. $\mathrm{N}_{\mathrm{a}}$ and $\mathrm{N}_{\mathrm{m}}$ are generated by zero-bias white noise routines which have been filtered (with a high-pass 
filter) to eliminate the noise components with a frequency $<0.35 \mathrm{~Hz}$. If the frequency is expressed as the number of turns per second inside the deployer, $0.35 \mathrm{turn} / \mathrm{s}$ corresponds to a tether exit speed of roughly $0.15 \mathrm{~m} / \mathrm{s}$ which is rarely encountered. The strategy of filtering the whiteband noise was adopted in order not to introduce unrealistic low-frequency-noise components which are better represented by the function $\mathrm{S}$.
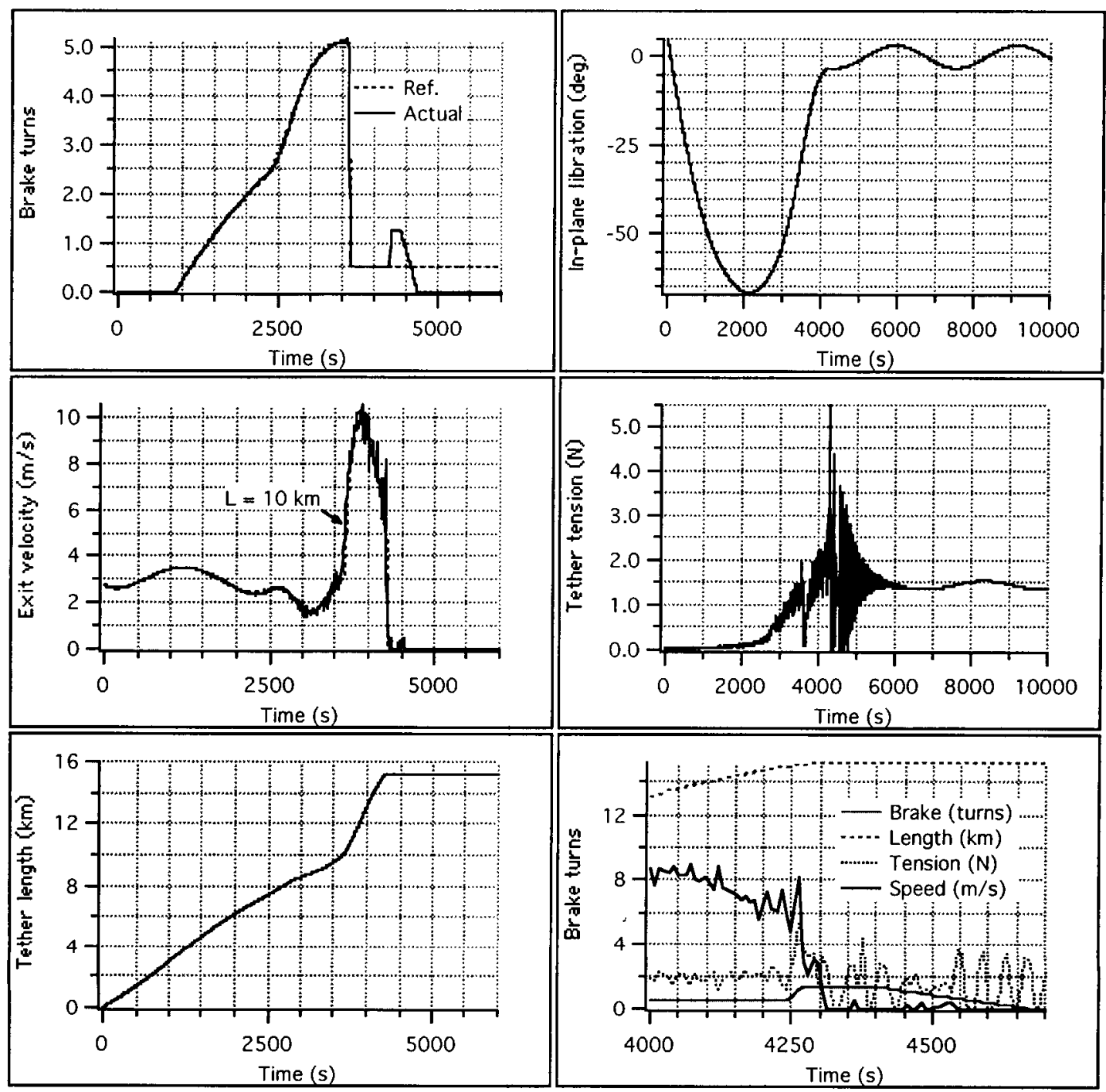

Fig. 5 Simulated deployment dynamics of ProSEDS with noise and $T_{0}=20 \mathrm{mN}$.

According to this model that is based on flight data, the tension is affected by additive and multiplicative white noise and a low-frequency (of about $0.5 \mathrm{~Hz}$ ) noise components. The additive noise component is important in the early phase of deployment when the tension is low and the tether deployment is dominated by the stick-slip mechanism (typically up to a tether length of about
$2-3 \mathrm{~km}$ ). The multiplicative term represents the increase of tension fluctuations proportionally to the instantaneous value of the tension which is dominant in the later stages of deployment and the low-frequency noise is due to the tether spooling.

Results of a deployment simulation with all the previously described noise component is shown in Fig. 5. The structure and intensity of the noise is as 
follows: (a) an additive noise component of $\pm 2 \mathrm{mN}$; and (b) a multiplicative noise component which fluctuates $\pm 50 \%$ with respect to the baseline value and a $0.5-\mathrm{Hz}$ sinusoidal component.

Since tension fluctuations influence the tether speed, the response to tension fluctuations is a test of the effectiveness of the filter in the control loop. In this particular control system which lacks a speedometer, the performance of the filter is especially important because the speed is computed from numerical derivation of the turn count and, consequently, it is very sensitive to noise affecting the turn count. The simulation results show that the filtering technique is very effective in abating the noise components.

Figure 6 shows the deployment trajectory of the endmass with respect to the Delta stage. The deployment time is marked by tickmarks at 6-min intervals. The overall deployment duration is 1 hour $18 \mathrm{~min}$ which includes the slow removal of the brake at the end of the maneuver.

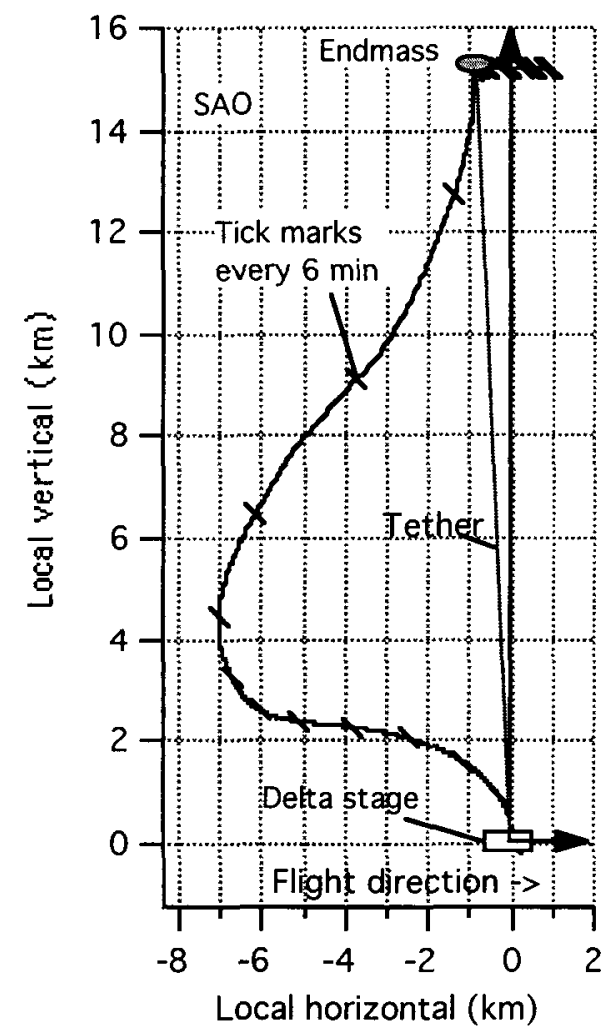

Figure 6 Deployment trajectory of endmass with respect to Delta stage.
These results and results from many other simulations (not shown here) demonstrate that the control law can tolerate large variations of the frictional parameters and also works well in the presence of noise.

\section{CONCLUSIONS}

The (closed-loop) control law of ProSEDS was developed with the goal of reducing the final libration amplitude to less than $20^{\circ}$ for a widerange variation of the key parameters. The control law was designed for robustness with respect to variations of the tension model parameters and of uncertainties in the brake response. Specifically, a variation of the most influential parameter, the minimum Dyneema tension, between $5 \mathrm{mN}$ and 30 $\mathrm{mN}$ is estimated to cause a final libration amplitude smaller than $8^{\circ}$. The minimum libration amplitude of $5^{\circ}$ is encountered for values of the minimum tether tension $T_{0}$ in the $15-20 \mathrm{mN}$ range. From simulation results, the control law also appears tolerant of tether tension affected by a high noise level.

Because of the present need to shorten the Dyneema tether by a few kilometers, the control law must be redesigned and retested while preserving the same control structure. The shortening of the tether segment, over which the feedback control can be exercised, will reduce the control authority. The challenge is to modify the control parameters and redesign the reference profile in such a way that the reduction in control capability is not too significant.

\section{ACKNOWLEDGMENTS}

This research has been supported by NASA Marshall Space Flight Center through Grant NAG8-1605 with Leslie Curtis as Technical Monitor.

\section{REFERENCES}

1 Pelaez, J., E.C. Lorenzini, O. Lopez-Rebollal, and M. Ruiz "A new kind of instability in electrodynamic tethers", The Journal of Astronautical Sciences, Vol. 48, No. 4, 449-476, 2000.

2 Lorenzini, E.C., S.B. Bortolami, C.C. Rupp, and F. Angrilli. "Control and Flight Performance of Tethered satellite Small expendable Deployment System-II" Journal of Guidance, Control and Dynamics, Vol. 19, No. 5, 1148-1156, 1996. 
3 Carroll, J. A., " Users Guide to SEDS, The Small Expendable-Tether Deployment System," Tether Applications, La Jolla, CA, April 1990.

4 Slotine, J.-J.E. and W. Li, "Applied Nonlinear Control." Prentice Hall, 1991.

5 Carroll, J.A., "SEDS Deployer Design and Flight Performance," Proceedings of the AIAA Space Programs and Technology Conference Exhibit, 21-23 September 1993, Huntsville, Alabama, AIAA 93-4764.

6 Nedler, J.A., and R. Mead, "A Simple Method for Function Minimization", Computer Journal, Vol. 7, pp. 308-313, 1965.

7 Palm III, W.J., "Modeling, Analysis and Control of Dynamics System." Wiley, 1983.

8 Lorenzini, E.C. et al., "Analytical Investigation of the Dynamics of Tethered Constellation in Earth Orbit (Phase II)." Smithsonian Astrophysical Observatory Final Report, NASA Contract NAS8-36606, May 1994. 